\title{
The specificity of the interaction with intestinal bacterial fermentation by prebiotics determines their physiological efficacy
}

\author{
Jan Van Loo \\ ORAFTI, Aandorenstraat 1, B3300 Tienen, Belgium
}

\begin{abstract}
The concept of prebiotic food ingredients is an important recent development in nutrition. The concept has attracted a great deal of attention, and many food ingredients (mainly dietary carbohydrates) have been claimed to be 'prebiotic'. It is emphasised that in order to be called prebiotic, a compound should be: (1) non-digestible; (2) fermentable; (3) fermentable in a selective way. These properties should be demonstrated in human volunteers in at least two independent dietary intervention trials. On the basis of published and unpublished results, it is shown in the present paper that the way in which a prebiotic influences intestinal fermentation is the key to its physiological properties. This statement is illustrated mainly by considering an established group of prebiotics, the $\beta(2-1)$ fructans. These linear molecules show a strong discontinuity in physicochemical properties as the chains become longer. The $\beta(2-1)$ fructans with a chain length of up to ten monomer units are very soluble and are particularly 'bifidogenic'. Longer chains (ten to sixty-five monomer units) are poorly soluble in water, they have less pronounced bifidogenic properties, and they are fermented more slowly. It was observed that a combination of short-chain and long-chain fructans (Synergy1) is physiologically (for example, increasing mineral absorption, suppressing carcinogenesis, modulating lipid metabolism, etc) more active than the individual fractions. A possible mechanism is described in the present review. From an in-depth overview of the literature it is confirmed that for prebiotic action, the 'selectivity principle' for intestinal fermentation is determinative for the type and for the efficiency of physiological activity. It is confirmed that prebiotics act through their influence on intestinal fermentation.
\end{abstract}

Prebiotics: Intestinal fermentation: Colonic bacteria: Inulin

\section{Introduction}

\section{Prebiotics}

Since the introduction of the term prebiotics (Gibson \& Roberfroid, 1995), a vast amount of literature related to the topic has been generated. Prebiotics have been defined as non-digestible food ingredients that affect the host beneficially by selectively stimulating the growth and/or activity of one or a limited number of bacteria in the colon, thus improving host health. As research has progressed, the definition has been fine-tuned.

There are three criteria that need to be fulfilled for a food ingredient to be designated a prebiotic.

First, the food ingredient must be non-digestible by host enzymes. Food ingredients need to be resistant to attack by digestive enzymes, such as carbohydrases from the brush border or those of pancreatic origin; as such, they remain available to the intestinal flora. The non-digestibility can be demonstrated by means of tests in vitro, using intestinal scrapings from various animals (pigs, rats, birds, fish). For human purposes, the best way to demonstrate non-digestibility is with ileostomised volunteers (Ellegard et al. 1997).

Second, the food ingredient must be fermented in the gastrointestinal (GI) tract. This can best be shown by means of fermentation tests in vitro. These tests show whether an ingredient is fermented, but in vitro models alone cannot be considered adequate for the study of the complex ecosystem of the colon (Newton et al. 1998).

Third, there must be selectivity in stimulation of the intestinal flora and of metabolic activity. This criterion is of

\footnotetext{
Abbreviations: ACF, aberrant crypt foci; DP, degree of polymerisation; $\mathrm{DP}_{\mathrm{av}}$, average degree of polymerisation; GI, gastrointestinal; HP-inulin, high-polymer inulin; OF, oligofructose; RS, resistant starch; SCFA, short-chain fatty acids; ST-inulin, native inulin; SYN1, Synergy1.

Corresponding author: Dr J. Van Loo, fax +32 16 801359, email Jan.Van.Loo@orafti.com
} 
utmost importance. A prebiotic ingredient, by definition, is not available to all bacteria of the intestinal ecosystem. Prebiotics must be particularly readily available to some groups of bacteria that are not related to a diseased state of the intestine (of which lactobacilli and bifidobacteria traditionally are considered indicator organisms). There are indications that this selectivity criterion originates in the genome of bacteria. Certain colonic bacteria may have specific transporters for certain types of prebiotics. This has been demonstrated with Lactobacillus acidophilus for oligofructose (OF; Barrangou et al. 2003). As a result, a prebiotic is less available to other groups of bacteria such as proteolytic bacteria, of which several are pathogenic. It is often the case that stimulating the growth of certain groups of bacteria allows them to take a more dominant position in the GI ecosystem, which they substantiate by producing metabolites, including bacteriocin-type products, or compounds related to quorum-sensing cascades.

A demonstration of the fulfilment of this criterion is much more difficult, and can be achieved only by means of repeated human dietary intervention studies. Whereas data from studies in vitro can give some mechanistic indications (production of metabolites such as short-chain fatty acids; SCFA), they cannot be fully representative of what happens in the human (or animal) GI environment. Data from experimental models cannot be extrapolated to man, as the GI architecture of different species of animals is dramatically different.

\section{Prebiotic activity}

The primary 'effect of prebiotics' (prebiosis) is an interaction with the GI ecosystem, which basically is an interaction with the fermentative capacity of the GI ecosystem. It is thought that all physiological effects that take place following prebiotic consumption originate in this altered fermentative functioning of the GI ecosystem. The initiation of physiological change originating in an altered fermentative capacity of the gut could happen through microbe-microbe interactions, host-microbe interactions and/or host-bacterial metabolite interactions. The latter may have the most important impact. The main metabolites are the SCFA: acetate, propionate and butyrate, which are all biologically active compounds (Cummings et al. 1995). Prebiotics can easily double the pool of SCFA in the GI tract, and the molar ratio between the different SCFA is altered (Djouzi \& Andrieux, 1997; Femia et al. 2002).

This process takes place in the intestine, having a surface of about $300 \mathrm{~m}^{2}$ in man, compared with $2 \mathrm{~m}^{2}$ for the skin or $100 \mathrm{~m}^{2}$ for the lungs. The lining of the GI tract is continuous with the external part of the body. As such, the surface of the intestine is the largest interface between our body and the environment. Via this large interface, we are in intimate contact with a very dense microbial ecosystem composed of up to $10^{12}$ bacteria/ml, which is, volumetrically, about the highest possible microbial population one can be in contact with. An improvement of the GI ecosystem thus represents an important improvement of the external environment in which we live. This improvement consists in a reduction of the numbers of pathogens and in relatively saccharolytic activity. Increased saccharolytic activity (endproducts are
SCFA, lactate and the gases $\mathrm{CO}_{2}$ and $\mathrm{H}_{2}$, which are ventilated via the breath and increased flatus) of the gut flora is concomitant with reduced proteolytic activity (production of putrefactive compounds and cytotoxic degradation products) because it is energetically more favourable to produce ATP from carbohydrates than from proteins. As long as carbohydrates are present, the bacteria need not establish a proteolytic catabolic enzyme apparatus, and hence less proteolysis takes place. It is thought that improving the composition of the intestinal ecosystem has a positive impact on the physiological functioning of an organism.

\section{Nutritional properties of prebiotics}

A direct consequence of prebiosis is an improved bowel habit. As prebiotic carbohydrates become available to the intestinal bacteria, they grow on it, increase in numbers and eventually in volume; they induce a faecal bulking effect. The SCFA products of fermentation stimulate intestinal peristalsis (Cherbut et al. 1991).

As a consequence of prebiotic consumption, there is a relief from constipation in chronically constipated individuals (Den Hond et al. 1997). As prebiotic carbohydrates arrive in the colon, they modify the composition of the intestinal flora, and they influence its metabolic activity. These processes increase the 'colonisation resistance'. This is the resistance of a certain established colonic bacterial population against other incoming bacteria (orally consumed) from finding a niche in the ecosystem (Newton et al. 1998). In a study with individuals travelling to 'highrisk' countries, it was observed that prebiotic consumption reduced the incidence of diarrhoea by almost $50 \%$ (Cummings et al. 2001).

It is thought that an increased production of propionate is at the basis of altered lipid metabolism. Propionate, which is produced from the fermentation of prebiotics, migrates via the portal vein to the liver, where it interacts with the regulation of the expression of genes that code for digestive hormones (incretins) such as glucagon-like peptide 1 and gastric inhibitory polypeptide, and of insulin (Kok et al. 1998).

The human studies that have been performed fail to show very uniform experimental outcomes. This is ascribed to the complexity of the biochemistry of lipid metabolism, which certainly complicates experimental protocols and outcomes (Van Loo et al. 1999; Pereira \& Gibson, 2002). Several studies in experimental models and in human dietary interventions, however, have shown that the parameters of lipid metabolism are not unaffected.

A consistent anticarcinogenic effect of dietary prebiotics has been observed in various experimental models.

The effect was shown in chemoprevention models in the short term (Reddy et al. 1997), where colonic aberrant crypt foci (ACF) are the biomarkers, as well as in longterm chemoprevention models (Verghese et al. 2002a,b) where the incidence of tumours is the biomarker.

In genetically predetermined Min-mice (apc-) models, there was a significant reduction of colonic tumours, and of tumours in the small intestine, indicating that systemic effects are involved (Pierre et al. 1997; M Lipkin, personal communication). 
The systemic efficacy was confirmed in models where tumour cells were implanted in muscle tissue (with growth rate of the tumour as a marker) or in peritoneum (with increase in life-span as a marker) (Taper et al. 1997, 1998).

At present, the anticancer effect of prebiotics in combination with probiotics is being investigated in a human volunteer study (Van Loo \& Jonkers, 2001).

The intake of prebiotics acidifies the intestinal contents, which solubilises minerals (mainly $\mathrm{Ca}$ or $\mathrm{Mg}$ salts) (Coudray et al. 2003b). The increased presence of butyrate, which is a selective source of energy for the intestinal epithelial cells, improves the absorptive capacity of the mucosa. Both these phenomena are thought to be at the basis of the repeatedly observed increase in mineral absorption from food in human volunteers (Coudray et al. 1997; van den Heuvel et al. 1998; Griffin et al. 2002).

\section{Goal of the present paper}

The present paper is intended to demonstrate that the particular way in which a prebiotic food ingredient interferes with the fermentation patterns of the GI ecosystem is central for improving the physiological functioning of the host. It will be shown that subtle changes in the chemical composition of the prebiotic food ingredient can have a major impact on the fermentation processes in the GI tract, which is reflected in an altered impact on certain physiological processes.

\section{The available evidence}

At present, there are only a few substrates for which a prebiotic action has been demonstrated adequately, i.e. that comply with all three prebiotic criteria as demonstrated by means of repeated human dietary intervention studies. These are the dietary oligosaccharides $\beta(2-1)$ fructans (inulins).

For the galacto-oligosaccharides there is strong evidence of selective stimulation of the intestinal flora; for isomaltooligosaccharides and xylo-oligosacchairdes, there is promising evidence. For the therapeutic disaccharide lactulose, there is good evidence.

As the inulins are dietary ingredients for which both prebiotic properties and effects on physiological function (mineral absorption, cancer experiments, immunological and lipid metabolism trials) have been demonstrated repeatedly in both experimental models and in human volunteers, they will be taken as the basis for further discussion.

\section{The influence of inulin on gastrointestinal fermentation}

Native inulin (ST-inulin) is obtained industrially from chicory roots by extraction with hot water, followed by refining and spray-drying. It is a polydisperse mixture of linear molecules, all with the same basic chemical structure, which is symbolised as $\mathrm{G}-\mathrm{F}_{n}(\mathrm{G}$, glycosyl moiety; F, fructosyl moiety; $n$, number of fructose units linked together through $\beta(2-1)$ bonds). The degree of polymerisation (DP) varies between three and sixty-five, and the average DP $\left(\mathrm{DP}_{\mathrm{av}}\right)$ is ten. OF, which is obtained by the partial enzymic hydrolysis of ST-inulin, is a mixture of molecules with chain lengths ranging between two and eight with a
$\mathrm{DP}_{\mathrm{av}}$ of four. High-polymer inulin (HP-inulin) is obtained by physically removing the lower-DP fraction from STinulin. The DP of HP-inulin ranges from ten to sixty-five, with a $\mathrm{DP}_{\mathrm{av}}$ of twenty-five. Synergy1 (SYN1) (Raftilose ${ }^{\circledR S y n e r g y} 1$ manufactured and commercialised for human consumption by ORAFTI NV, Tienen, Belgium) is a 1:1 mixture of OF and HP-inulin (see Fig. 1).

The bifidogenicity of each of those fractions of inulin has been demonstrated repeatedly in human dietary intervention studies (Roberfroid et al. 1998; Van Loo et al. 1999). From these reviews, it appears that the short-chain inulin (OF) increases the bifidobacteria by almost an order of magnitude more (order of magnitude 1 log colony-forming unit) than the HP-inulins (order of magnitude $0 \cdot 1 \mathrm{log}$ colony-forming unit), but selectivity has been demonstrated in both cases (Tuohy et al. 2001; Harmsen et al. 2002). The bifidobacteria function as indicator organisms of a well-balanced GI flora in terms of increased saccharolytic activity relative to proteolytic activity, which is monitored by quantifying the SCFA:branched SCFA ratio (Swanson et al. 2002). It is obvious that the OF has more impact on improving the composition of the microflora (relative amounts of different groups of GI bacteria) than the other fractions.

There is a remarkable physicochemical property of the inulin chains that is central in the explanation of differences in the way different fractions influence GI fermentation.

Whereas a DP range of three to sixty-five is relatively small, there is a big difference in the solubility of inulin chains with different lengths. Those with a DP from three to ten are very soluble (up to $85 \%(\mathrm{w} / \mathrm{v})$ in water at room temperature), while molecules with a chain length of ten to sixty-five are rather poorly soluble (up to $2 \%(\mathrm{w} / \mathrm{v})$ in water at room temperature). It is thought that this is related to the thermodynamic behaviour of inulin in solution, of which various metastable forms exist (Marchessault, 1980).

It has been shown that the in vitro faecal slurry fermentation of the short-chain inulins or OF occurs very quickly; $1 \%(\mathrm{w} / \mathrm{v})$ at $\mathrm{pH} 6.7$ and $37^{\circ} \mathrm{C}$ with $2 \%(\mathrm{w} / \mathrm{v})$ inoculum is completely fermented in $5 \mathrm{~h}$. However, the fermentation of the long chains or HP-inulin takes about three to five times as long (Roberfroid et al. 1998; Baeten, 1999).

It has been shown (J Van Loo, unpublished data) that long-chain inulin is fermented completely in the GI tract of human subjects consuming $10 \mathrm{~g} / \mathrm{d}$ or more (none is recovered in faeces). Because the long chains are fermented slowly, they reach more distal regions in the colon, where they can maintain a selective metabolic activity (Tuohy et al. 2001; Harmsen et al. 2002). Whereas HP-inulin modifies the composition of the intestinal flora significantly but to a limited extent as compared with $\mathrm{OF}$, it is transformed into biologically active metabolites for a longer period of time and in more distal regions of the intestine.

The hypothesis behind the formulation of SYN1 was to provide a short-chain fraction that is able to modify the composition of the colonic flora. This necessarily occurs in the proximal part of the colon, given its rapid fermentation. The remaining fraction, HP-inulin, functions subsequently as a slow but selective substrate for the modified GI ecosystem. As a direct consequence, the improved GI ecosystem can exert its beneficial effects for a longer period of time; 


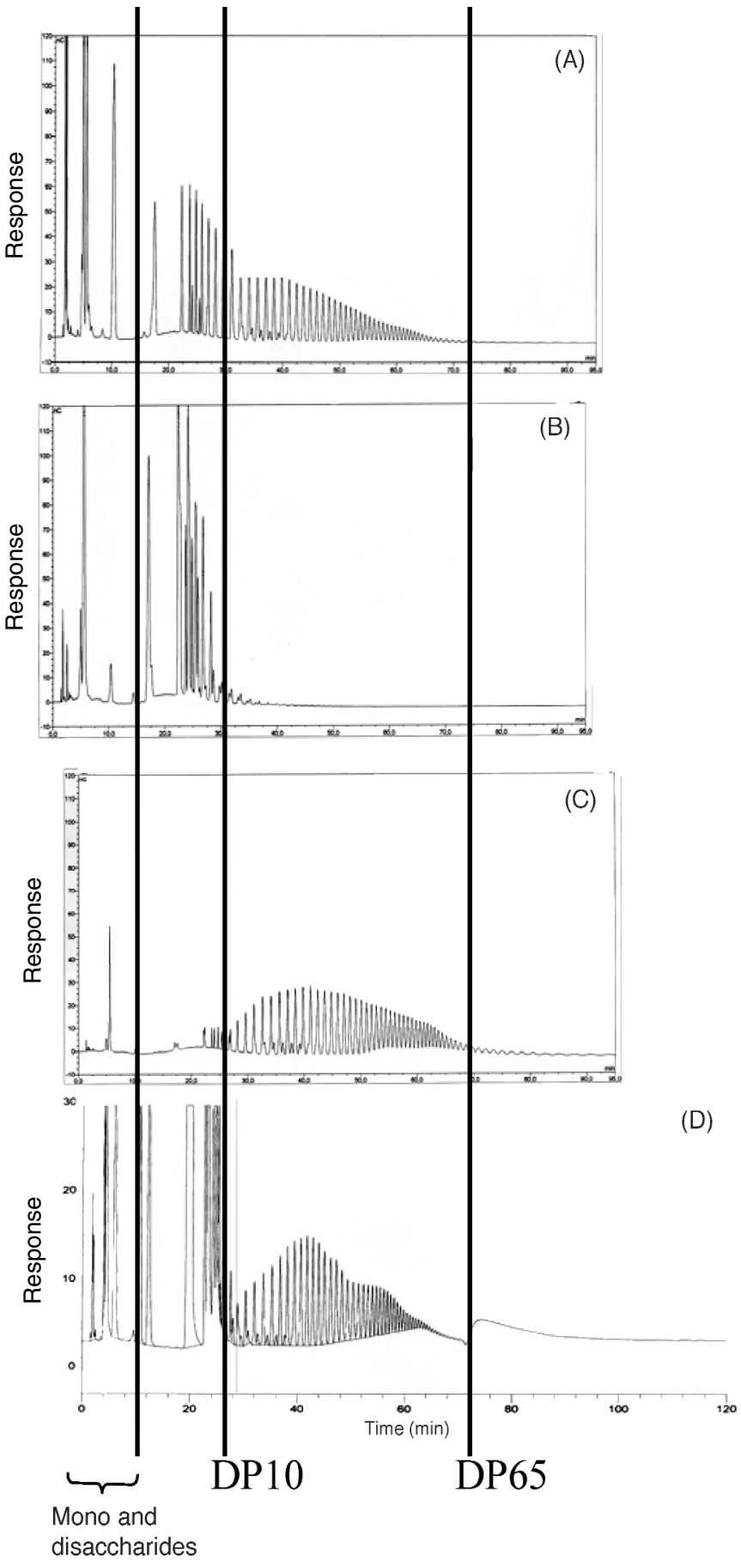

Fig. 1. High-performance, anion-exchange chromatograms (obtained with a pulsed amperometric detector; Van Loo et al. 1995) of the different chicory inulin fractions that were tested in this work. The technique is not quantitative, but shows accurately the distribution of various chain lengths. The monosaccharides are fructose and glucose; the disaccharide is sucrose. ST-inulin (A) is the inulin as extracted from the chicory root. The degree of polymerisation (DP) varies from three to sixty-five; the average $D P\left(D P_{\text {av }}\right)$ is ten. Oligofructose $(\mathrm{B})$ is partially hydrolysed ST-inulin (DP between two and eight; $\mathrm{DP}_{\mathrm{av}}$ 4). HP-inulin (C) is ST-inulin devoid of the oligofructose fraction (DP between ten and 65; $\mathrm{DP}_{\mathrm{av}} 25$ ). Synergy1 (D) is a 1:1 mixture of oligofructose and HP-inulin (ORAFTI NV, Tienen, Belgium). an implicit consequence is that the improved GI ecosystem can extend its metabolic activity into more distal areas of the GI tract. Until now it has been impossible to demonstrate these phenomena in situ and in vivo but, as will be seen later, there are indirect indications of the validity of the hypothesis.

So far, the observation and interpretation of the prebiotic properties of inulin reveals that subtle differences in the molecular structure (chain length) of carbohydrates can have a major impact on fermentation processes.

The fact that non-digestible carbohydrates with different molecular composition and binding properties have dramatically different influences on fermentation has been demonstrated (Wang \& Gibson, 1993; Gibson et al. 1995; Hopkins et al. 1998). For inulin there is at present a vast set of experimental evidence from both fermentation and dietary intervention studies. Therefore the rest of the present paper will focus on inulin. It is emphasised that this does not imply that other prebiotics could not contribute to the discussion. There however is no evidence available to date. The present discussion on inulin allows us to show the impact of prebiotics that only differ in one aspect (chain length). All other chemical parameters are similar (composing monosaccharide, type of binding), which makes the discussion from this point of view scientifically more relevant.

\section{Impact of the type of fermentation (inulin fraction) on physiological processes}

\section{Anticarcinogenesis studies}

The anticarcinogenic properties of inulin have been demonstrated by means of various experimental models (chemoprevention, genetically predetermined mouse model and tumour implantation models; Van Loo \& Jonkers, 2001). In the chemoprevention studies, the effect of inulin fractions with different chain length has been studied (Verghese et al. 2003). The idea was to replace some of the starch (10\% with no effect on the growth rate of the animals) in a standard rat diet with OF, ST-inulin, HP-inulin and SYN1.

It was observed that the greater the proportion of chains with DP of ten to sixty-five (series OF (0\% DP > 10); STinulin (50\% DP > 10); HP-inulin (100\% DP > 10)), the smaller the number of total aberrant crypts observed in the colon. From Fig. 2, it appears that at the same time the ACF with high multiplicity (three, four, and five and more ACF/focus) decreases markedly.

The observation with SYN1 (50\% DP > 10) is not in accord with these observations; whereas the relative amount of long chains is similar to ST-inulin, its effect on the reduction of ACF is decreased markedly. It may be observed from Fig. 2 that the reduction of foci with high multiplicity is much more pronounced for SYN1 than for the other inulin fractions.

Fig. 3, which is derived from the same experiment, shows that the higher the fraction with DP $>10$, the more important becomes the effect of inulin in the distal part of the colon (series OF (0 \% DP > 10); ST-inulin (50\% DP > 10); HP-inulin (100\% DP > 10)). Again, there is a disparity 


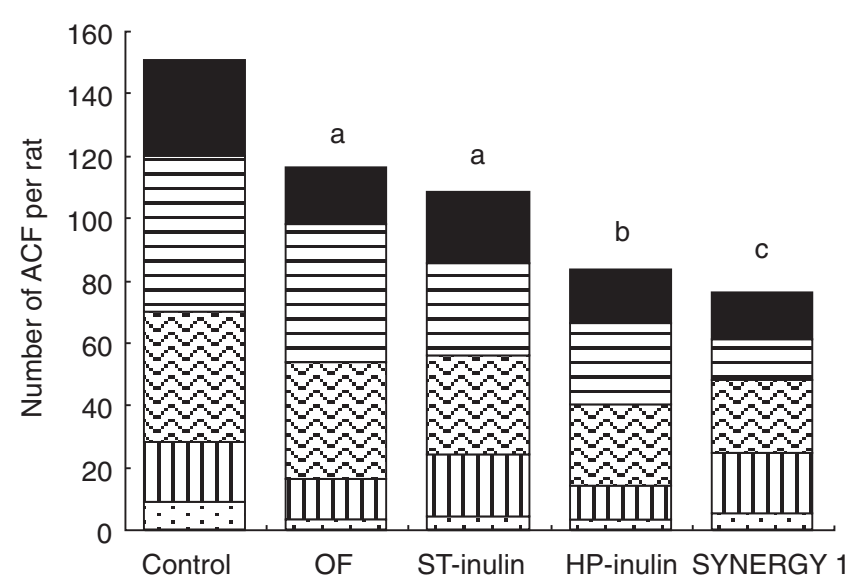

Fig. 2. A chemoprevention trial in which rats $(n 12)$ were challenged twice (1-week interval) with azoxymethane (15 mg/kg body weight). They were given the respective diets (control; oligofructose (OF); native inulin (ST-inulin); high-polymer inulin (HP-inulin); Synergy1 (1:1 mixture of OF and HP-inulin; ORAFTI NV, Tienen, Belgium)) 2 weeks before carcinogen application, and then for 8 weeks more, after which the aberrant crypt foci (ACF) were counted, and classified as foci with one, two and up to five ACF per focus. The more ACF per focus, the higher the probability of development into a tumour. ( $\square$ ), Five or more ACF/focus; ( $\boxminus$ ), four ACF/focus; $(\mathrm{G})$, three ACF/focus; (П), two ACF/focus; (- $\cdot$ ), one ACF/focus. $a, b, c$ Values with unlike superscript letters are significantly different; all are significantly different from the control $(P<0.05)$ (from Verghese et al. 2003).

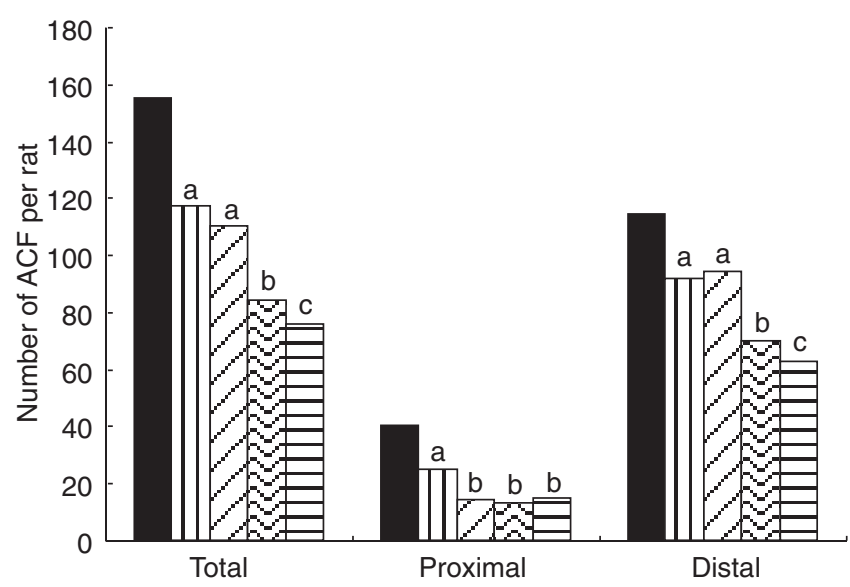

Fig. 3. Data from the same experiment as in Fig. 2, but rearranged to show the effects in the proximal and distal colon. The diets were: control ( $\square$ ), oligofructose (OF; $\square$ ), native inulin (ST-inulin; $\square$ ), high-polymer inulin (HP-inulin; $\mathrm{A})$, and Synergy 1 (1:1 mixture of OF and HP-inulin; ORAFTI NV, Tienen, Belgium) $(\square)$ It is observed that short chains (OF) exert beneficial effects particularly in the proximal colon. The higher the proportion of long chains, the more important becomes the anticarcinogenic effect in the distal part of the colon (series ST-inulin and HP-inulin). There is a smaller proportion of long chains in Synergy1 than in HP-inulin, but the additional effect on the composition of the flora in the proximal colon induced by the short-chain fraction of Synergy1 (see Fig. 1) could explain the difference. ${ }^{\text {a,b.c }}$ Values with unlike superscript letters in each group (total, proximal, distal) are significantly different; all are significantly different from the control (from Verghese et al. 2003). in this observation with SYN1, where the effect in the distal colon is more pronounced than with any of the other fractions (Fig. 3).

This difference can be explained by the additional effect of SYN1; i.e. the modification (with its short-chain fraction) of the flora in the proximal colon to a greater degree than the other prebiotic food ingredients. This would allow the fermentation of its long-chain fraction (DP $>10)$ to occur by a more potent anticarcinogenic intestinal population. Also, the long-chain fraction (DP > 10) in SYN1 is, on average, longer ( $\left.\mathrm{DP}_{\mathrm{av}} 25\right)$ than in ST-inulin $\left(\mathrm{DP}_{\mathrm{av}} 14\right)$, but is similar to that in HP-inulin ( $\left.\mathrm{DP}_{\mathrm{av}} 25\right)$.

Earlier, Reddy et al. (1997) reported a difference between OF and HP-inulin in a similar protocol, which these authors attributed to a difference in fermentation rate between $\mathrm{OF}$ and HP-inulin, as the carcinogen azoxymethane induces ACF in more distal parts of the colon. As long chains are fermented more slowly, the observed effect could be an indirect demonstration that they also migrate to more distal parts in the colon, where they can maintain a more saccharolytic (production of SCFA) fermentation.

Klinder et al. (2003) quantified cellular parameters of survival (differentiation, tumour progression, and invasive growth) in HT29 cells and Caco 2 cells, which they challenged with supernatant fractions of fermentation broths originating from a three-stage colon simulation model. They compared a fermentation broth that had glucose as a C-source with a fermentation broth that had SYN1 as the C-source. It was observed that SYN1 fermentation broths reduced the markers of tumour progression significantly more than the glucose fermentation broth.

Glucose is a commonly available C-source. The microflora developed on glucose is different from that established on SYN1. The different effect of the supernatant fractions of both on the molecular processes taking place in the tested cell lines demonstrates that fermentation pattern (bacteria and metabolites) plays a central role in the influence that these exert on physiological processes.

Conclusion. The results of these experiments suggest that the modification of the gut microflora is key in causing the anticarcinogenic effect of inulin. Slowly and selectively fermented long chains, however, sustain the effect (selectively maintain the metabolic activity), which is reflected in a stronger physiological impact (ACF reduction in this case).

It is observed that the longer the chain length, the smaller the number of crypts with high multiplicity.

Whereas the fraction of long chains is smaller in SYN1, there are markedly fewer ACF with high multiplicity than are seen with HP-inulin. The additional effect of the shortchain fraction on the intestinal flora in the proximal part of the colon could explain the difference.

The type of fermentation determines how the physiological processes are influenced.

\section{Studies on increased mineral absorption}

Experimental models. The increase of mineral absorption ( $\mathrm{Ca}, \mathrm{Mg}$ ) from food by the dietary administration of inulin or 
its fractions to experimental models (Delzenne et al. 1995; Ohta et al. 1998; Coudray et al. 2003b) and human volunteers (Coudray et al. 1997; van den Heuvel et al. 1999; Griffin et al. 2002; Cashman, 2003; L Holloway, S Moynihan and A Friedlander, unpublished results) is well documented.

Reconsidering these studies with an emphasis on chain length, or on the fermentation properties of the particular inulin types used, can contribute to the goal of the present paper.

In an exploratory mineral absorption experiment, Delzenne et al. (1995) used ST-inulin and OF as test substances. They observed significant increases in the retention of $\mathrm{Ca}, \mathrm{Mg}$ and $\mathrm{Zn}$ and they did not observe significant differences in efficacy between OF and ST-inulin.

Recently, Coudray et al. (2003b) reported the results of a study where the effect of OF, HP-inulin and SYN1 on Ca and $\mathrm{Mg}$ absorption were compared with a control (no nondigestible oligosaccharides added). They confirmed the results reported by Delzenne et al. (1995) for OF for both $\mathrm{Ca}$ and $\mathrm{Mg}$ absorption. They observed additionally that HPinulin was as efficient as OF with respect to increased $\mathrm{Ca}$ absorption, but that it increased $\mathrm{Mg}$ absorption significantly more than OF. The effect of SYN1 in this experiment was significantly more efficient in increasing $\mathrm{Ca}$ absorption from the diet than both OF and HP-inulin. There was no additional effect on $\mathrm{Mg}$ absorption, but this may be due to saturation, as over $75 \%$ of all dietary $\mathrm{Mg}$ is absorbed (Fig. 4).

In the experimental models on mineral absorption, there seems to be a trend similar to that seen in the anticarcinogenesis studies. The more slowly fermented longer chains increase the length of time of the physiological observation. In the mineral absorption model, however, the effect of the 1:1 OF:HP-inulin combination product is markedly more pronounced than in the anticancer models.

Whereas the caecal soluble Ca pool was increased 20- to 30 -fold in all inulin-type fed groups, the increased solubility of $\mathrm{Ca}$ and $\mathrm{Mg}$ did not correlate with the increased mineral absorption capacity of the different inulin types. There was no observable difference in caecal SCFA composition. This suggests that the difference in improving physiological properties of the different inulin fractions is taking place more distally than the caecum, presumably in the colon. Ohta et al. (1998) showed an increase of calbindin in the caecum and colorectum. It should be investigated whether long chains in HP-inulin or SYN1 affect calbindin presence in the colorectal area more than OF does.

The difference in the Ca increase between the SYN1 diet and the OF diet is much greater than the difference between the HP-inulin diet and the OF diet. This suggests that the combination of rapidly fermented prebiotics (OF chains in caecum) and slowly fermented prebiotics (HP-inulin chains in colon and rectum) enhances mineral absorption significantly.

Younes et al. (2001) published the results of a study in rats where ST-inulin, resistant starch (RS) and a combination of the two were compared. They observed that both ST-inulin (+61 \%) and RS (+34\%) increased Ca absorption, and that the combination of the two increased $\mathrm{Ca}$ absorption significantly more (+130\%; from 23 to $53 \%$ absorption of the dietary $\mathrm{Ca}$ ) than their component units.
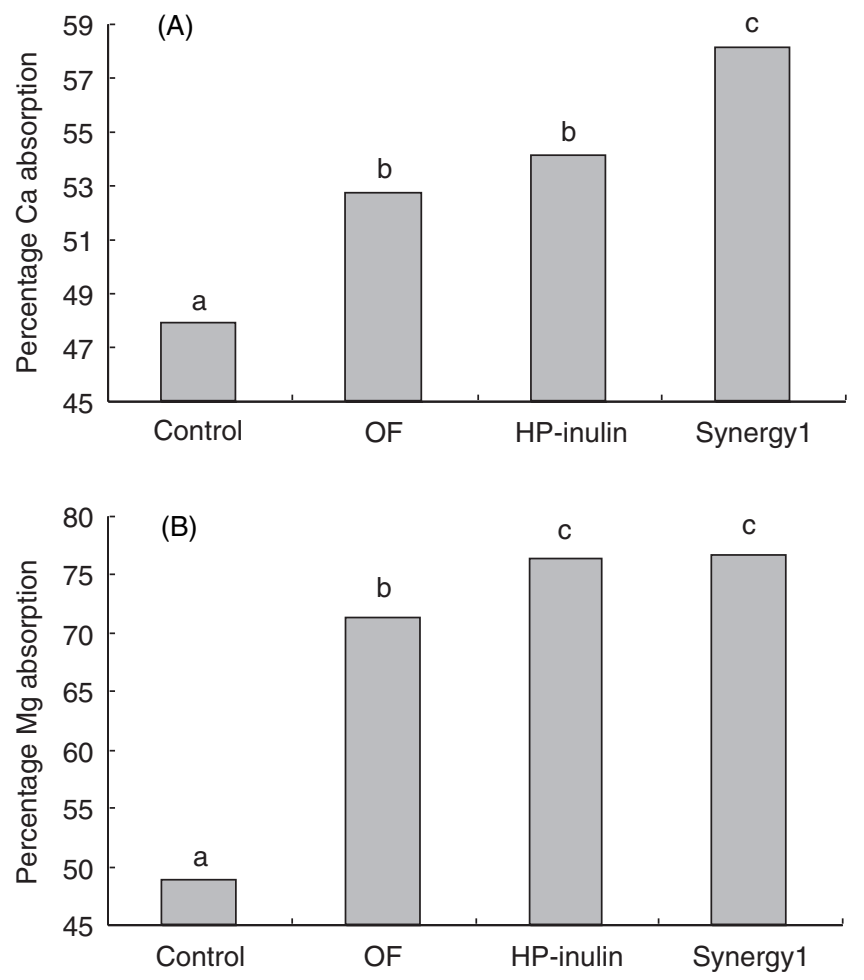

Fig. 4. Increase of percentage $\mathrm{Ca}$ absorption (A) and percentage $\mathrm{Mg}$ absorption (B) upon intake of $10 \%$ oligofructose (OF), highpolymer inulin (HP-inulin) and Synergy 1 (1:1 mixture of OF and HPinulin; ORAFTI NV, Tienen, Belgium) by rats. The presence of long chains increases the mineral absorption, but for Synergy 1 there is a markedly more important effect on $\mathrm{Ca}$ absorption. ${ }^{\mathrm{a}, \mathrm{b}, \mathrm{c}}$ Values with unlike superscript letters are significantly different $(P<0.05)$ (from Coudray et al. 2003b).

All three diets increased Mg absorption significantly but there was no significant additional effect due to the combination.

There was significantly more caecal SCFA with the RS diet and the combination diet than there was with the STinulin diet (and in all three cases significantly more than in control; about 3-fold). RS had significantly more SCFA produced than ST-inulin, but the percentage Ca absorption was (not significantly) lower with RS. This suggests that SCFA and other fermentative factors play a role (other metabolites, types of bacteria that are stimulated).

Human dietary intervention studies. Griffin et al. (2002) recently published data that contribute to the present discussion. They used a double-blind, placebo-controlled, cross-over study design with two cohorts of thirty volunteers (girls 11-14 years), who were given a high intake of $\mathrm{Ca}(1500 \mathrm{mg} / \mathrm{d})$ in order to be above the saturation level $(1200 \mathrm{mg} \mathrm{Ca} / \mathrm{d})$. This was done to avoid the possibility that varying sub-optimal $\mathrm{Ca}$ intakes would interfere with the results. One group of volunteers was given SYN1 at a dose of $8 \mathrm{~g} / \mathrm{d}$ and another group received OF at $8 \mathrm{~g} / \mathrm{d}$. The percentage $\mathrm{Ca}$ absorption from the food was measured with a dual-stable-isotope technique, each group against its own control. 
The results are presented in Fig. 5, which shows clearly that under the experimental conditions of very high $\mathrm{Ca}$ intake, SYN1 but not OF increases Ca absorption significantly.

In previously published studies with OF at $15 \mathrm{~g} / \mathrm{d}$ for $9 \mathrm{~d}$ (van den Heuvel et al. 1999) or with ST-inulin at up to 40 $\mathrm{g} / \mathrm{d}$ during 1 month (Coudray et al. 1997) it was shown that both have the ability to increase $\mathrm{Ca}$ absorption, albeit at higher levels of intake.

Tahiri et al. (2001) have observed an increased $\mathrm{Mg}$ absorption of $12 \%$ but no effect on Ca absorption (Tahiri et al. 2003) with short-chain fructo-oligosaccharides (a synthetic equivalent of OF) $(n 11 ; 10 \mathrm{~g} / \mathrm{d}$ for a period of 5 weeks in a cross-over study design) in post-menopausal women. Similar results with other non-digestible oligosaccharides (of which prebiotic activity has not been proven to date) such as maltitol and hydrogenated maltitol also increased $\mathrm{Mg}$ absorption, but did not affect $\mathrm{Ca}$ absorption (Coudray et al. 2003a).

In a similar population of post-menopausal women, (L Holloway, S Moynihan and A Friedlander, unpublished results) have observed a significant increase in both $\mathrm{Mg}$ absorption of $22 \%$ and a significant increase in Ca absorption of $39 \%$ with SYN1 $(n 15 ; \mathrm{SYN} 1$ at $10 \mathrm{~g} / \mathrm{d}$ for a period of 6 weeks in a double-blind, placebo-controlled cross-over study design).

Conclusion. Both experimental models and human dietary intervention studies show clearly that there is a link
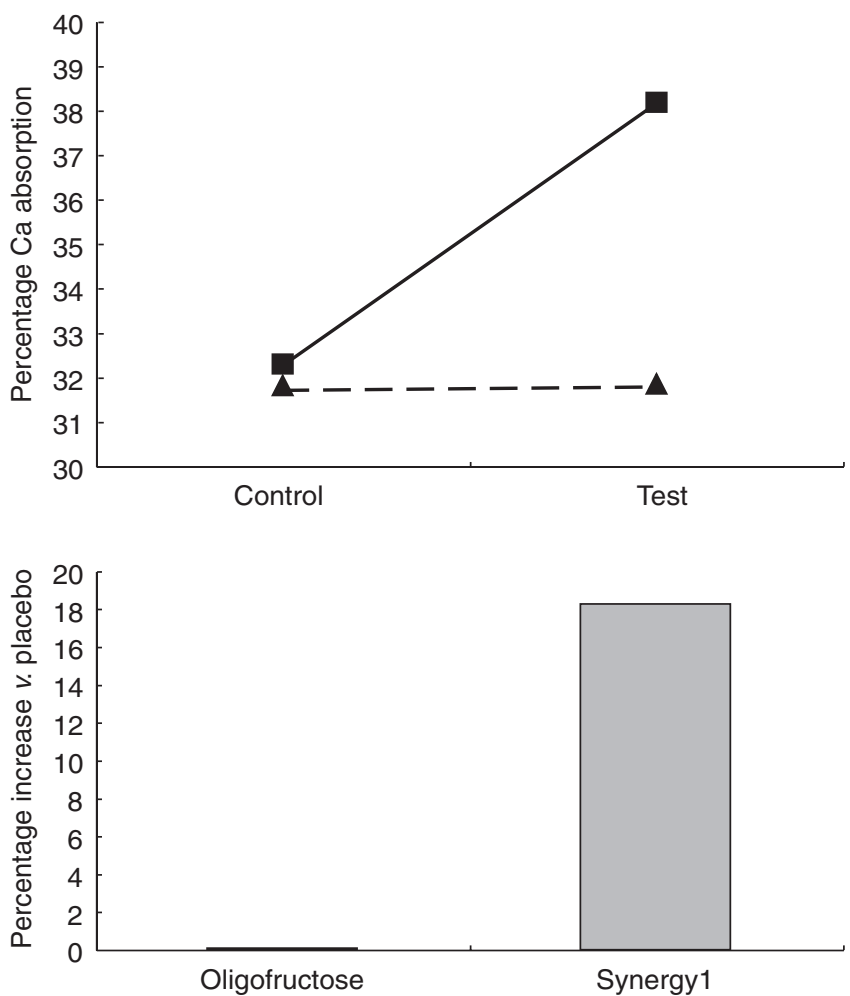

Fig. 5. Under conditions of high intake levels of $\mathrm{Ca}(1500 \mathrm{mg} / \mathrm{d})$, Synergy1 (1:1 mixture of oligofructose and high-polymer inulin; ORAFTI NV, Tienen, Belgium) ( $\boldsymbol{\square})$ but not oligofructose $(\boldsymbol{\Delta})$ is able to increase significantly $(P<0.05)$ the dietary percentage $\mathrm{Ca}$ absorption as measured by dual-stable-isotope techniques (from Griffin et al. 2002). between the observed increase in mineral absorption and the type of prebiotic used. As a consequence, there is a link between the GI fermentation pattern of a prebiotic carbohydrate and the effect on mineral absorption.

From the experiments, it follows that the effect increases with increasing chain length, which, in the case of inulin, means slower selective fermentation.

In mineral absorption studies too, the effect of the combination of a rapidly and selectively fermented substrate (OF) with a slowly and selectively fermented substrate (HP-inulin) is significantly more effective in increasing $\mathrm{Ca}$ absorption than the inulin fractions it is composed of.

This indicates that there is a positive interaction between the effects on mineral absorption of a rapid fermentation, which results in highly significant changes in the composition of intestinal flora, and the effects of slow fermentation, which may keep the modified flora active for a longer period of time and probably into more distal parts of the intestine.

\section{Studies on immunology}

Buddington et al. (2002) fed mice a control diet, a diet with $10 \%$ HP-inulin and a diet containing $10 \%$ OF. After 2 weeks adaptation, a group of animals were injected intraperitoneally with Listeria monocytogenes and another group with Salmonella typhimurium, in such a dose as to cause about a $50 \%$ mortality after a certain period of time. For the Listeria challenge, the authors observed a significant decrease in mortality in the OF-fed animals, but for the HP-inulin the effect was significantly more pronounced, to even $0 \%$ mortality. For Salmonella, there was no significant numerical decrease in mortality for the OF-fed animals, but the effect of HP-inulin remained significant (Fig. 6). In the Listeria-challenged mice, there was a significantly increased natural killer cell activity of the splenocytes as well as a significantly higher phagocytic activity of peritoneal macrophages (Kelly-Quagliana et al. 2003). Whereas the difference between OF and HP-inulin was not significant, both values were numerically more important in $\mathrm{HP}$-inulin than in OF.

SYN1 was not available at the time these experiments were carried out.

Conclusion. Even in systemic physiological processes such as the immune system, the effect of different fermentation patterns of the prebiotic exerts its influence. The more slowly fermented long-chain HP-inulin seems to be more efficient in promoting effects than the short chains of OF.

\section{Studies on factors related to lipid metabolism}

Here, the data are less consistent. Some published human dietary intervention studies show effects on cholesterol, others on serum triacylglycerol levels, others on both and still other studies show no effect (Van Loo et al. 1999; Pereira \& Gibson, 2002). From the several studies published, three were performed with OF (Alles et al. 1997; van Vliet, 1997; van Dokkum et al. 1999) and had no effect on the levels of cholesterol or serum triacylglycerols. Four were carried out with ST-inulin, of which three had an 

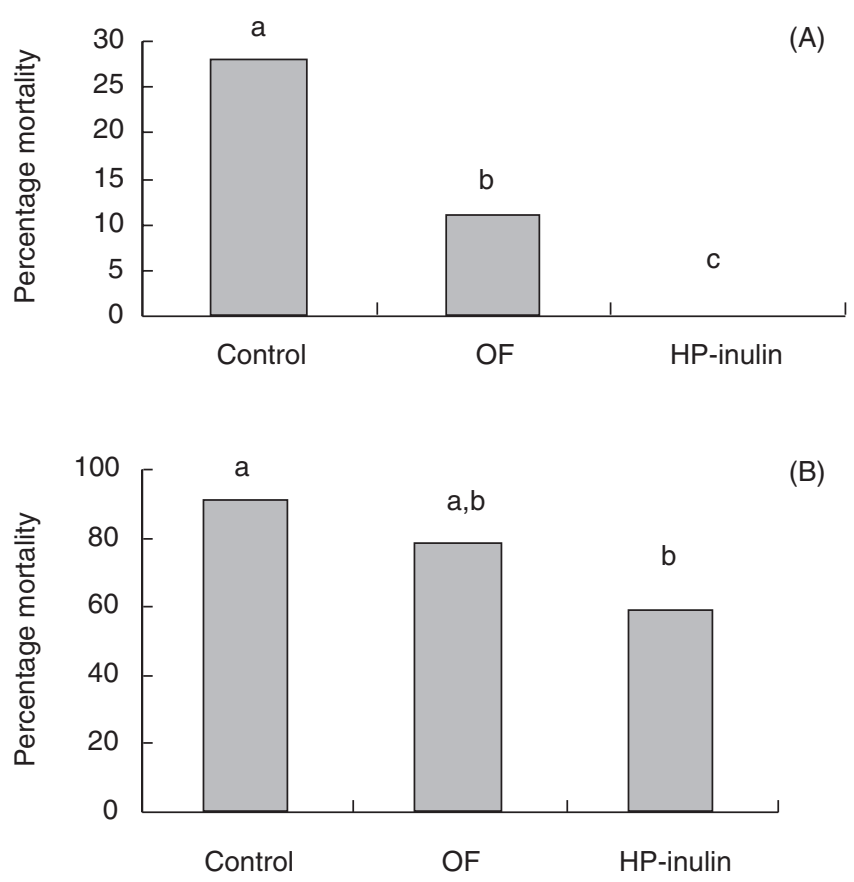

Fig. 6. In challenge experiments (Listeria monocytogenes $(\mathrm{A})$ and Salmonella typhimurium (B)) where the model was fed either an oligofructose (OF)- or a high-polymer-inulin (HP-inulin)-containing diet, the percentage mortality was significantly different from the control. In the Listeria challenge, HP-inulin was significantly more active than OF, and this was the case numerically in the Salmonella challenge. ${ }^{a, b, c}$ Values with unlike superscript letters are significantly different $(P<0.05)$ (from Buddington et al. 2002).

effect on at least one of the measured parameters of lipid metabolism (Pedersen et al. 1997; Brighenti et al. 1999; Davidson \& Maki, 1999) and one had no effect (van Dokkum et al. 1999). Two studies were performed with HP-inulin and both were effective in affecting parameters of lipid metabolism. One showed significant effects on serum triacylglycerols and on insulin (Jackson et al. 1999), and one showed clear effects on hepatic lipogenesis (Letexier et al. 2003).

Conclusion. In none of these studies was the effect of two types of inulin compared in the same protocol. It therefore cannot be claimed on a solid basis that long chains are more efficient than short chains in affecting lipid metabolism. The observation that studies performed with long chains (ST-inulin and HP-inulin) gave more positive results than the studies performed with OF seem to confirm the trend described earlier.

\section{General discussion and conclusion}

Prebiotics selectively modify the composition and/or the metabolic activity of the GI ecosystem, which is the external environment with which the human body is in closest contact. The way in which a prebiotic interacts with that environment depends very much on its molecular structure.

There were significant effects on mineral absorption, cancer risk reduction, and immunology with prebiotics that typically modify the flora but do not maintain the fermentation (rapidly fermented prebiotics such as OF). However, there seems to be a positive correlation between the effect on all physiological fields of interest mentioned, including lipid metabolism, and the presence of long chains.

There is a clear link between molecular structure, GI fermentation and physiological efficacy. This relationship is more complex than a simple dose-effect relationship, as the size of impact of the latter is very much dependent on the initial status (bacteriological and physiological) of a volunteer. This issue has been discussed by Roberfroid et al. (1998).

From these observations, it can be concluded that it is the type of GI fermentation that finally affects the physiological observations.

This hypothesis is confirmed by experiments with combination products composed of a rapidly and selectively fermented substrate (flora composition effect, physiological effects) and a slowly fermented substrate (sustained maintenance of GI flora metabolism). The physiological impact of such a combination product is more important than that of its components. This illustrates the adjuvant effect of 'flora composition' and 'sustained metabolic maintenance', and hence that impact on GI fermentation causes the physiological observations.

In other words it can be claimed that prebiotics, through their impact on GI fermentation, influence physiological processes, most of which are considered biomarkers for health status.

\section{References}

Alles MS, De Roos NM, Backx JC, van de Lisdonk E \& Hautvast J (1997) Consumption of fructo-oligosaccharides does not affect blood glucose and serum lipids in patients with type 2 diabetes. American Journal of Clinical Nutrition, 69, 64-69.

Baeten J (1999) Invloed van inuline-type fructanen op de darmflora : simulaties door middel van in vitro batchfermentaties met fecale slurry (On the influence of inulin-type fructans on the intestinal flora simulations by means of in vitro batch fermentations with faecal slurry). Thesis Vrije Universiteit Brussel, Brussels, Belgium.

Barrangou R, Altermann E, Hutkins R, Cano R \& Klaenhammer TR (2003) Functional and comparative genomic analyses of an operon involved in fructooligosaccharide utilization by Lactobacillus acidophilus. Proceedings of the National Academy of Sciences USA 100, 8957-8962.

Brighenti F, Casiraghi MC, Canzi E \& Ferrari A (1999) Effect of consumption of a ready-to-eat breakfast cereal containing inulin on the intestinal milieu and blood lipids in healthy male volunteers. European Journal of Clinical Nutrition 53, 726-733.

Buddington KK, Donahoo JB \& Buddington RK (2002) Dietary oligofructose and inulin protect mice from enteric and systemic pathogens and tumor inducers. Journal of Nutrition 132, 472-477.

Cashman K (2003) Prebiotics and calcium bioavailability. Current Issues of Intestinal Microbiology 4, 21-32.

Cherbut C, Salvador V, Barry J-LDF \& Delort-Laval J (1991) Dietary fibre effects on intestinal transit in man: involvement of their physicochemical and fermentative properties. Food Hydrocolloids 5, 15-22.

Coudray C, Bellanger J, Castiglia-Delavaud C, Remesy C, Vermorel M \& Rayssiguier Y (1997) Effect of soluble or partly soluble dietary fibres supplementation on absorption and 
balance of calcium, magnesium, iron and zinc in healthy young men. European Journal of Clinical Nutrition 51, 375-380.

Coudray C, Bellanger J, Vermorel M, Sinaud S, Wils D, FeilletCoudray C, Brandolini M, Bouteloup-Demange C \& Rayssiguier Y (2003a) Two polyol, low digestible carbohydrates improve the apparent absorption of magnesium but not of calcium in healthy young men. Journal of Nutrition 133, 90-93.

Coudray C, Tressol JC, Gueux E \& Rayssiguier Y (2003b) Effects of inulin-type fructans of different chain length and type of branching on intestinal absorption and balance of calcium and magnesium in rats. European Journal of Nutrition 42, 91-98.

Cummings JH, Christie S \& Cole T (2001) A study of fructooligosaccharides in the prevention of travellers' diarrhoea. Alimentary Pharmacology and Therapeutics 15, 1139-1145.

Cummings JH, Rombeau JL \& Sakata T (1995) Physiological and Clinical Aspects of Short-Chain Fatty Acids. Cambridge, UK: Cambridge University Press.

Davidson MH \& Maki KC (1999) Effects of dietary inulin on serum lipids. Journal of Nutrition 129, 1474S-1477S.

Delzenne N, Aertssens J, Verplaetse H, Roccaro M \& Roberfroid M (1995) Effect of fermentable fructo-oligosaccharides on mineral, nitrogen and energy digestive balance in the rat. Life Sciences 57, 1579-1587.

Den Hond EM, Geypens BJ \& Ghoos YF (1997) Effect of long chain chicory inulin on bowel habit and transit time in constipated persons. Nutrition Research 20, 731-736.

Djouzi Z \& Andrieux C (1997) Compared effects of three oligosaccharides on metabolism of intestinal microflora in rats inoculated with a human faecal flora. British Journal of Nutrition 78, 313-324.

Ellegard L, Andersson H \& Bosaeus I (1997) Inulin and oligofructose do not influence the absorption of cholesterol, or the excretion of cholesterol, $\mathrm{Ca}, \mathrm{Mg}, \mathrm{Zn}, \mathrm{Fe}$, or bile acids but increases energy excretion in ileostomy subjects. European Journal of Clinical Nutrition 51, 1-5.

Femia AP, Luceri C, Dolara P, Giannini A, Biggeri A, Salvadori M, Clune Y, Collins KJ, Paglierani M \& Caderni G (2002) Antitumorigenic activity of the prebiotic inulin enriched with oligofructose in combination with the probiotics Lactobacillus rhamnosus and Bifidobacterium lactis on azoxymethaneinduced colon carcinogenesis in rats. Carcinogenesis 23, 1953-1960.

Gibson GR, Beatty ER, Wang X \& Cummings JH (1995) Selective stimulation of bifidobacteria in the human colon by oligofructose and inulin. Gastroenterology 108, 975-982.

Gibson GR \& Roberfroid MB (1995) Dietary modulation of the human colonic microbiota: introducing the concept of prebiotics. Journal of Nutrition 125, 1401-1412.

Griffin IJ, Davila PM \& Abrams SA (2002) Non-digestible oligosaccharides and calcium absorption in girls with adequate calcium intakes. British Journal of Nutrition 87, Suppl. 2, S187-S191.

Harmsen H, Raangs G, Franks A, Wildeboeer-Veloo A \& Welling $G$ (2002) The effect of the prebiotic inulin and the probiotic Bifidobacterium longum on the fecal microflora of healthy volunteers measured by FISH and DGGE. Microbial Ecology in Health and Disease 14, 211-219.

Hopkins M, Cummings J \& Macfarlane G (1998) Inter-species differences in maximum specific growth rates and cell yield of bifidobacteria cultured on oligosaccharides and other simple carbohydrate sources. Journal of Applied Microbiology 85, 381-386.

Jackson KG, Taylor GR, Clohessy AM \& Williams CM (1999) The effect of the daily intake of inulin on fasting lipid, insulin and glucose concentrations in middle-aged men and women. British Journal of Nutrition 82, 23-30.
Kelly-Quagliana KA, Nelson PD \& Buddington RK (2003) Dietary oligofructose and inulin modulate immune functions in mice. Nutrition Research 23, 257-267.

Klinder A, Gietl E, Hughes R, Jonkers N, Karlsson P, McGlyn H, Pistoli S, Tuohy K, Rafter J, Rowland I, Van Loo J \& PoolZobel B (2003) Gut fermentation products of chicory inulinderived prebiotics inhibit markers of tumor progression in human colon tumor cells. Proceedings of the 2nd meeting of Frontiers in Cancer Prevention Research.

Kok NN, Morgan LM, Williams CM, Roberfroid MB, Thissen JP \& Delzenne NM (1998) Insulin, glucagon-like peptide 1, glucose-dependent insulinotropic polypeptide and insulin-like growth factor I as putative mediators of the hypolipidemic effect of oligofructose in rats. Journal of Nutrition 128, 1099-1103.

Letexier D, Diraison F \& Beylot M (2003) Addition of inulin to a moderately high-carbohydrate diet reduces hepatic lipogenesis and plasma triacylglycerol concentrations in humans. American Journal of Clinical Nutrition 77, 559-564.

Marchessault R (1980) Conformation and crystalline structure of (2-1)-beta-D-fructofuranan (inulin). Canadian Journal of Chemistry 58, 2415-2422.

Newton D, Cummings JH, Macfarlane S \& Macfarlane G (1998) Growth of a human intestinal Desulfovibrio desulfuricans in continuous cultures containing defined populations of saccharolytic and amino acid fermenting bacteria. Journal for Applied Microbiology 85, 371-380.

Ohta A, Motohashi Y, Ohtsuki M, Hirayama M, Adachi T \& Sakuma K (1998) Dietary fructooligosaccharides change the concentration of calbindin-D9k differently in the mucosa of the small and large intestine of rats. Journal of Nutrition 128, 934-939.

Pedersen AA, Sandstrom B \& Van Amelsvoort JMM (1997) The effect of ingestion of inulin on blood lipids and gastrointestinal symptoms in healthy females. British Journal of Nutrition $\mathbf{7 8}$ $215-222$.

Pereira DI \& Gibson GR (2002) Effects of consumption of probiotics and prebiotics on serum lipid levels in humans. Critical Reviews in Biochemistry and Molecular Biology 37, 259-281.

Pierre F, Perrin P, Champ M, Bornet F, Meflah K \& Menanteau J (1997) Short-chain fructo-oligosaccharides reduce the occurrence of colon tumors and develop gut-associated lymphoid tissue in Min mice. Cancer Research 57, 225-228.

Reddy BS, Hamid R \& Rao CV (1997) Effect of dietary oligofructose and inulin on colonic preneoplastic aberrant crypt foci inhibition. Carcinogenesis 18, 1371-1374.

Roberfroid MB, Van Loo JA \& Gibson GR (1998) The bifidogenic nature of chicory inulin and its hydrolysis products. Journal of Nutrition 128, 11-19.

Swanson KS, Grieshop CM, Flickinger EA, Bauer LL, Wolf BW, Chow JM, Garleb KA, Williams JA \& Fahey GC (2002) Fructooligosaccharides and Lactobacillus acidophilus modify bowel function and protein catabolites excreted by healthy humans. Journal of Nutrition 132, 3042-3050.

Tahiri M, Tressol JC, Arnaud J, Bornet F, Bouteloup-Demange C, Feillet-Coudray C, Ducros V, Pepin D, Brouns F, Rayssiguier AM \& Coudray C (2001) Five-week intake of short-chain fructo-oligosaccharides increases intestinal absorption and status of magnesium in postmenopausal women. Journal of Bone Mineralisation Research 16, 2152-2160.

Tahiri M, Tressol JC, Arnaud J, Bornet FR, Bouteloup-Demange C, Feillet-Coudray C, Brandolini M, Ducros V, Pepin D, Brouns F, Roussel AM, Rayssiguier Y \& Coudray C (2003) Effect of shortchain fructooligosaccharides on intestinal calcium absorption and calcium status in postmenopausal women: a stable-isotope study. Amercian Journal of Clinical Nutrition 77, 449-457. 
Taper HS, Delzenne NM \& Roberfroid MB (1997) Growth inhibition of transplantable mouse tumors by non-digestible carbohydrates. International Journal of Cancer Research $\mathbf{7 1}$, 1109-1112.

Taper HS, Lemort C \& Roberfroid MB (1998) Inhibition effect of dietary inulin and oligofructose on the growth of transplantable mouse tumor. Anticancer Research 18, 4123-4126.

Tuohy K, Finlay RK, Wynne AG \& Gibson GR (2001) A human volunteer study on the prebiotic effects of HP-inulin - faecal bacteria enumerated using fluorescent in situ hybridisation (FISH). Anaerobe 7, 113-118.

van den Heuvel EG, Muys T, van Dokkum W \& Schaafsma G (1999) Oligofructose stimulates calcium absorption in adolescents. American Journal of Clinical Nutrition 69, 544-548.

van den Heuvel EG, Schaafsma G, Muys T \& van Dokkum W (1998) Nondigestible oligosaccharides do not interfere with calcium and nonheme-iron absorption in young, healthy men. American Journal of Clinical Nutrition 67, 445-451.

van Dokkum W, Wezendonk B, Srikumar TS \& van den Heuvel EG (1999) Effect of nondigestible oligosaccharides on largebowel functions, blood lipid concentrations and glucose absorption in young healthy male subjects. European Journal of Clinical Nutrition 53, 1-7.

Van Loo J, Cummings J, Delzenne N, Englyst H, Franck A, Hopkins M, Kok N, Macfarlane G, Newton D, Quigley M, Roberfroid M, van Vliet T \& van den Heuvel E (1999) Functional food properties of non-digestible oligosaccharides: a consensus report from the ENDO project (DGXII AIRIICT94-1095). British Journal of Nutrition 81, 121-132.

Van Loo J \& Jonkers N (2001) Evaluation in human volunteers of the potential anticarcinogenic activities of novel nutritional con- cepts: prebiotics probiotics and synbiotics (the SYNCAN project). Nutrition and Metabolic Cardiovascular Disease 11, 87-93.

Van Loo J, Coussement P, De Leenheer L, Hoebregs H \& Smits G (1995) On the presence of inulin and oligofructose as natural ingredients in the western diet. Critical Reviews in Food Science and Nutrition 35, 525-552.

van Vliet T (1997) A Double Blind Placebo Controlled, Parallel Trial on the Effect of Oligofructose Intake on Serum Lipids in Male Volunteers. TNO Report V 97.874. Zeist, The Netherlands: TNO.

Verghese M, Rao DR, Chawan CB \& Shackelford L (2002a) Dietary inulin suppresses azoxymethane-induced preneoplastic aberrant crypt foci in mature Fisher 344 rats. Journal of Nutrition 132, 2804-2808.

Verghese M, Rao DR, Chawan CB, Williams LL \& Shackelford L (2002b) Dietary inulin suppresses azoxymethane-induced aberrant crypt foci and colon tumors at the promotion stage in young Fisher 344 rats. Journal of Nutrition 132, 2809-2813.

Verghese M, Walker LT, Shackelford L, Chawan CB \& Van Loo J (2003) Inhibitory effects of non-digestible carbohydrates of different chain lengths on AOM-induced aberrant crypt foci in Fisher 344 rats. Proceedings of the 2nd meeting of Frontiers in Cancer Prevention Research.

Wang X \& Gibson GR (1993) Effects of the in vitro fermentation of oligofructose and inulin by bacteria growing in the human large intestine. Journal of Applied Bacteriology 75, 373-380.

Younes H, Coudray C, Bellanger J, Demigne C, Rayssiguier Y \& Remesy C (2001) Effects of two fermentable carbohydrates (inulin and resistant starch) and their combination on calcium and magnesium balance in rats. British Journal of Nutrition $\mathbf{8 6}$, 479-485. 